\title{
Management of difficult-to-treat patients with ulcerative colitis: focus on adalimumab
}

This article was published in the following Dove Press journal:

Drug Design, Development and Therapy

5 April 2013

Number of times this article has been viewed

\section{Alessandro Armuzzi \\ Daniela Pugliese \\ Olga Maria Nardone \\ Luisa Guidi}

IBD Unit, Complesso Integrato Columbus, Catholic University, Rome, Italy
Correspondence: Alessandro Armuzzi Complesso Integrato Columbus, Catholic University, Via G. Moscati 3I-3300I68 Rome, Italy

$\mathrm{Tel}+39063503310$

Fax +3906305 464I

Email alearmuzzi@yahoo.com

\begin{abstract}
The treatment of ulcerative colitis has changed over the last decade, with the introduction of biological drugs. This article reviews the currently available therapies for ulcerative colitis and the specific use of these therapies in the management of patients in different settings, particularly the difficult-to-treat patients. The focus of this review is on adalimumab, which has recently obtained approval by the European Medicines Agency and the US Food and Drug Administration, for use in treating adult patients with moderate-to-severe, active ulcerative colitis, who are refractory, intolerant, or who have contraindications to conventional therapy, including corticosteroids and thiopurines. Since the results emerging from the pivotal trials have been subject to some debate, the aim of this review was to summarize all available data on the use of adalimumab in ulcerative colitis, focusing also on a retrospective series of real-life experiences. Taken together, the current evidence indicates that adalimumab is effective for the treatment of patients with different types of ulcerative colitis, including biologically naïve and difficult-to-treat patients.
\end{abstract}

Keywords: randomized controlled trials, real-life experiences

\section{Introduction}

Ulcerative colitis is a chronic inflammatory disease of the large intestine. Its exact cause is unknown, but it appears to be multifactorial, with a proposed interaction between genetic and environmental factors that results in continuous activation of the intestinal mucosal immune system. The inflammation affects the mucosa of the rectum, with different degrees of involvement of the colon. ${ }^{1}$ The management of ulcerative colitis is based on the extent of colon involvement, the activity, and the behavior of disease. The classification of the disease is defined according to the Montreal Classification, which describes the maximal macroscopic extent of the disease at colonscopy. ${ }^{2}$ This has important prognostic and management implications because patients with extensive ulcerative colitis bear a higher risk of colectomy and cancer, ${ }^{3}$ and patients with proctitis and left-sided colitis obtain much more benefit from topical therapies. However, the extent of colon involvement may change over time, such that about $20 \%$ of patients who are diagnosed with proctitis or left-sided ulcerative colitis are found to have proximal extension of the inflammation at follow-up.

The clinical course of ulcerative colitis is characterized by different disease onsets and a remitting-relapsing course. A recent population-based, inception cohort study identified four different patterns: (1) initial high activity that decreases to remission or mild severity (55\% of patients); (2) initial low activity that changes to increased severity ( $1 \%$ of patients); (3) continuous symptoms ( $6 \%$ of patients); and (4) chronic 
intermittent symptoms (37\% of patients). Moreover, an initial presentation with extensive colitis, high systemic inflammation burden, and a younger age has been associated with higher subsequent colectomy rates. ${ }^{4}$ Disease activity is commonly classified as: remission, mild, moderate, or severe. Over the years, several different scoring systems have been developed as measures of disease activity, but most of these have only been used in clinical trials and have not been validated. In clinical practice, the combination of clinical features, laboratory findings, and the endoscopic appearance, forms the basis of patient management. ${ }^{5}$

\section{Management of ulcerative colitis}

The main treatment goals for ulcerative colitis are the induction and maintenance of clinical and endoscopic remission. As far as mild-to-moderate disease is concerned, the oral and topical aminosalicylates represent the standard therapy for achieving this outcome. ${ }^{6}$ In the event of inadequate response to aminosalicylates and in patients with moderate-to-severe disease, systemic corticosteroids are the best option for inducing remission. ${ }^{7}$ Patients with active ulcerative colitis who do not have significant clinical improvement after 2-4 weeks of an appropriate course of corticosteroids are classified as "corticosteroid-refractory." Anti-tumor necrosis factor-alpha (TNF $\alpha$ ) monoclonal antibodies represent the best available option for this group of patients, achieving clinical and endoscopic remission without prolonged steroid exposure. ${ }^{8}$

After achieving a response with corticosteroid treatment, aminosalicylates are usually continued as maintenance therapy. However, patients relapsing within 3 months of stopping corticosteroids or who are not able to reduce the dose to below $10 \mathrm{mg} /$ day of prednisolone within 3 months of starting are classified as "corticosteroid dependent." In this specific disease setting, azathioprine has been shown to be significantly more effective than mesalazine for inducing corticosteroidfree clinical and endoscopic remission at 6 months and has a corticosteroid-sparing effect. ${ }^{9}$ Treatment algorithms for patients with corticosteroid-dependent ulcerative colitis suggest starting concomitant thiopurine therapy and slowly withdrawing corticosteroids over 3-4 months, timed to coincide with the expected onset of action of the thiopurines. ${ }^{10}$ If symptoms persist or patients are unable to stop steroids after 12 weeks of starting thiopurines, anti-TNF $\alpha$ agents should be started. ${ }^{10}$ Finally, induction and scheduled maintenance treatment with infliximab has been recently reported to be effective for inducing steroid-free clinical remission and mucosal healing at 1 year, in both thiopurine-naïve and experienced, corticosteroid-dependent, ulcerative colitis patients. ${ }^{11}$
Patients with acute, severe ulcerative colitis need to be hospitalized and treated with intensive intravenous corticosteroids (methylprednisolone, $60 \mathrm{mg} / 24 \mathrm{~h}$, or hydrocortisone, $100 \mathrm{mg}$, four times daily). A lack of improvement within 3-5 days of intensive treatment is an indication for rescue therapy or surgery. A recent open-label trial involving 115 patients with acute, severe ulcerative colitis who were refractory to intravenous corticosteroids and randomized to receive either intravenous cyclosporine or infliximab has shown no significant differences in treatment failure (primary efficacy outcome: $60 \%$ cyclosporine group vs $54 \%$ infliximab group; absolute risk difference, 6\%; 95\% confidence interval $[\mathrm{CI}],-7$ to $19[P=0.52]) .^{12}$

Therefore, the decisions of physicians are often determined on a case-by-case basis. These decisions are usually made based on personal experiences with this specific therapy and the physician's confidence for the management of adverse events, taking into account the long-term strategy. Cyclosporine, in fact, has been shown to be effective only over the short-to-medium term. Therefore, all patients should be bridged to thiopurines, although it has been shown that patients without previous thiopurine exposure have better outcomes. $^{13}$

\section{Adalimumab in ulcerative colitis}

Adalimumab is a human monoclonal immunoglobulin (Ig) G1 antibody to TNF $\alpha$ that is subcutaneously administered at a standard induction dose of $160 \mathrm{mg}$, followed by $80 \mathrm{mg}$ after 2 weeks. Maintenance doses are then scheduled at $40 \mathrm{mg}$ every other week (EOW). ${ }^{14}$ This drug has been shown to be effective for inducing and maintaining remission in patients with active, moderate-to-severe luminal or perianal Crohn's disease; patients naïve to anti-TNF $\alpha$; or patients with previous loss of response or intolerance to infliximab. ${ }^{15-19}$

As far as ulcerative colitis is concerned, after the publication of the results of the two pivotal, randomized, placebocontrolled, double-blind trials (ULTRA 1 and 2) (Table 1), ${ }^{20,21}$ adalimumab was approved for use in patients with moderateto-severe active disease and in those who were nonresponders or intolerant to conventional therapy. In these trials, involving more than 1000 patients with moderate-to-severe active ulcerative colitis, adalimumab was compared with placebo with regard to the efficacy of induction and as a maintenance treatment, assessed after 8 and 52 weeks, respectively.

In the ULTRA 1 trial, ${ }^{20}$ patients with ulcerative colitis were initially randomized to adalimumab (160 mg/80 mg) or placebo at weeks 0 and 2, respectively. Subsequently, after an amendment of the protocol, a third arm, with adalimumab 
Table I Outcome parameters from studies on adalimumab in ulcerative colitis

\begin{tabular}{|c|c|c|c|c|c|c|c|}
\hline $\begin{array}{l}\text { Study } \\
\text { (references) }\end{array}$ & $\begin{array}{l}\text { Study } \\
\text { type }\end{array}$ & $\begin{array}{l}\text { Number } \\
\text { of patients }\end{array}$ & $\begin{array}{l}\text { Induction of clinical } \\
\text { response/remission } \\
\text { (\%) }\end{array}$ & $\begin{array}{l}\text { Maintenance of clinical } \\
\text { response/remission } \\
\text { (\%) }\end{array}$ & $\begin{array}{l}\text { Steroid } \\
\text { sparing } \\
(\%)\end{array}$ & $\begin{array}{l}\text { Mucosal } \\
\text { healing } \\
(\%)\end{array}$ & $\begin{array}{l}\text { Colectomy } \\
\text { (\%) }\end{array}$ \\
\hline ULTRA I20 & RCT & 390 & Week 8: 54.6\%/18.5\% & - & - & $\begin{array}{l}\text { Week 8: } \\
46.9 \%\end{array}$ & - \\
\hline ULTRA $2^{21}$ & RCT & 494 & Week 8: 50.4\%/I6.5\% & Week 52: 34.6\%/I7.3\% & $37.8 \%$ & $\begin{array}{l}\text { Week 8: } \\
41.1 \% \\
\text { Week 52: } \\
25 \%\end{array}$ & - \\
\hline $\begin{array}{l}\text { Oussalah } \\
\text { et } \mathrm{al}^{27}\end{array}$ & OL & 13 & - & $\begin{array}{l}42 \text { weeks mean FU: } \\
38.5 \% \text { clinical benefit }\end{array}$ & - & - & $46.2 \%$ \\
\hline Afif et $\mathrm{a}^{28}$ & OL & 20 & Week 8: 25\%/5\% & Week 24: 50\%/25\% & $58 \%$ & - & - \\
\hline Hudis et $\mathrm{a}^{29}$ & RS & 9 & - & - & $56 \%$ & - & - \\
\hline Gies et al ${ }^{10}$ & OL & 25 & Week I4: $80 \% /-$ & $\begin{array}{l}54.5 \text { weeks median FU: } \\
70 \% /-\end{array}$ & $100 \%$ & - & $8 \%$ \\
\hline $\begin{array}{l}\text { Taxonera } \\
\text { et al }\left.\right|^{31}\end{array}$ & RS & 30 & Week I2: 60\%/26.7\% & $\begin{array}{l}48 \text { weeks median FU: } \\
50 \% /-\end{array}$ & $68 \%$ & - & $20 \%$ \\
\hline $\begin{array}{l}\text { Ferrante } \\
\text { et } \mathrm{al}^{33}\end{array}$ & RS & 50 & Week 4: 68\%/- & $\begin{array}{l}23 \text { months median FU: } \\
52 \% /-\end{array}$ & - & - & $20 \%$ \\
\hline $\begin{array}{l}\text { McDermott } \\
\text { et a }\left.\right|^{32}\end{array}$ & RS & 23 & - & $\begin{array}{l}23 \text { months median FU: } \\
35 \% \text { clinical benefit }\end{array}$ & - & & \\
\hline $\begin{array}{l}\text { García-Bosch } \\
\text { et al }{ }^{34}\end{array}$ & RS & 48 & Week I2: 70.8\%/50\% & Week 54: 35\%/30\% & - & - & $22.9 \%$ \\
\hline $\begin{array}{l}\text { Armuzzi } \\
\text { et } \mathrm{a}^{35}\end{array}$ & RS & 88 & Week I2: $-/ 28.4 \%$ & Week 54: $-143.2 \%$ & $56.7 \%$ & $26.3 \%$ & $25 \%$ \\
\hline
\end{tabular}

Abbreviations: RCT, randomized controlled trial; OL, open-label study; RS, retrospective study; FU, follow-up.

at $80 \mathrm{mg} / 40 \mathrm{mg}$, was included. All patients enrolled were naïve to anti-TNF $\alpha$ therapy and had active disease (defined by a full Mayo score of 6-12 and an endoscopic subscore of 2-3), despite stable doses of concomitant steroids, immunomodulators, or both. The primary endpoint, assessed in 390 patients with ulcerative colitis who were studied after the above amendment, was defined as the proportion of patients achieving clinical remission (full Mayo score $\leq 2$, with no individual subscore $>1$ ) by week 8 in each treatment arm. Week 8 clinical remission was achieved in $18.5 \%$ of patients in the adalimumab $160 / 80 \mathrm{mg}$ group and in $9.2 \%$ of patients in the placebo arm $(P=0.031)$, showing a $9.3 \%$ of therapeutic gain. The week 8 clinical remission rate in the adalimumab $80 / 40 \mathrm{mg}$ group was similar to that of the placebo group ( $10 \%$ vs $9.2 \%)(P=0.833)$. The clinical response and mucosal healing among the three groups (secondary endpoints) were not significantly different. A post hoc analysis identified baseline clinical variables, such as extensive disease, high disease activity (Mayo score $\geq 10$ ) and high levels of systemic inflammation (C-reactive protein $=10 \mathrm{mg} / \mathrm{L}$ ), that were associated with a low proportion of patients in clinical remission, which might reflect a lesser efficacy of adalimumab in patients with more severe disease. Thereafter, 390 patients entered an open-label extension study after week 8 and were maintained on adalimumab
$40 \mathrm{mg}$ EOW for 52 weeks, with the possibility of doseescalation to $40 \mathrm{mg}$ weekly. A clinical remission at week 52 was reported in $25.6 \%$ of patients maintained with $40 \mathrm{mg}$ of adalimumab EOW. A post hoc analysis, which included the patients who dose-escalated to $40 \mathrm{mg}$ weekly, showed that $29.5 \%$ of patients were in remission at week $52 .{ }^{22}$

In the ULTRA 2 trial, 494 active ulcerative colitis patients were randomized to receive adalimumab $160 \mathrm{mg}$ at week $0,80 \mathrm{mg}$ at week 2, and $40 \mathrm{mg}$ EOW, or placebo, through to 52 weeks. The clinical and endoscopic eligibility characteristics were similar to those associated with the ULTRA 1 study, with the exception of the inclusion of ulcerative colitis patients ( $40 \%$ of the population studied) who had already experienced anti-TNF $\alpha$ agents, but with a discontinuation period of at least 8 weeks. The two coprimary endpoints were defined as the proportion of patients achieving clinical remission (defined as full Mayo score $\leq 2$, with no individual subscore $>1$ ) at week 8 and the proportion of patients achieving clinical remission at week 52. Clinical remission at week 8 was achieved in $16.5 \%$ of patients in the adalimumab arm and in $9.3 \%$ of patients in the placebo $\operatorname{arm}(P=0.019)(7.2 \%$ therapeutic gain). The corresponding values at week 52 were $17.3 \%$ and $8.5 \%(P=0.004)$, respectively, with an absolute difference of adalimumab versus placebo of $8.8 \%$. Moreover, a clinical response was 
achieved in $50.4 \%$ of patients receiving adalimumab and $34.6 \%$ on placebo $(P<0.001)$ at week 8 and in $30.2 \%$ and $18.3 \%$, respectively $(P=0.002)$ at week 52 . The benefit over placebo was also significant by endoscopic remission, evaluated at week 8 (41.1\%, adalimumab vs $31.7 \%$, placebo) $(P=0.032)$ and at week $52(25 \%$ vs $15.4 \%$, respectively $)$ $(P=0.009)$. A subgroup analysis, stratifying patients based on prior exposure to anti-TNF $\alpha$, was also performed. Among naïve patients, a week 8 clinical remission was achieved in $21.3 \%$ of patients in the adalimumab group and in $11 \%$ in the placebo group $(P=0.017)$; the corresponding values at week 52 were $22 \%$ and $12.4 \%$, respectively $(P=0.029)$. A significant difference in clinical remission was found only at week $52(10.2 \%$, adalimumab and $3 \%$, placebo) $(P=0.039)$ in the anti-TNF $\alpha$-exposed group. ${ }^{21}$

A post hoc intention-to-treat analysis of ULTRA 2, including all patients randomized to adalimumab who achieved a clinical response, as per their partial Mayo score at week 8, was performed to investigate week 52 clinical remission, response, mucosal healing, corticosteroid-free remission, and corticosteroid discontinuation rates. Among the 248 patients originally randomized to adalimumab, $123(49.6 \%)$ had achieved clinical response. Of these, 30.9\%, $49.6 \%$, and $43.1 \%$ achieved clinical remission, clinical response, and mucosal healing at week 52, respectively. Of the 150 adalimumab-treated patients taking corticosteroids at enrollment, 90 (60\%) responded, as per their partial Mayo score at week 8 . Of these, $21.1 \%$ achieved corticosteroid-free remission and $37.8 \%$ were corticosteroid-free at week 52 , without significant differences among the anti-TNF $\alpha$-naïve and exposed patients. These results were similar whether or not week 8 responses were assessed using the full Mayo score. ${ }^{23}$ Further analysis showed that patients who received the $160 \mathrm{mg} / 80 \mathrm{mg}$ adalimumab induction dose had a significantly lower risk of all-cause hospitalizations and ulcerative colitis-related hospitalizations, compared with placebo, during the first 8 weeks of therapy. ${ }^{24}$ This benefit over placebo was also significant for adalimumab early-responders, during the follow-up. ${ }^{25}$

At 52 weeks, 588 patients who completed the ULTRA 1-2 trials entered an extension, open-label study. Patients who entered the open-label, weekly adalimumab study continued at the same dose. Patients who entered the study from any blinded arm or from an open-label cohort receiving adalimumab (40 mg EOW) received adalimumab at a dose of $40 \mathrm{mg}$ EOW. At week 60 of the open-label extension study, 351 (59.7\%) of the patients who had entered the extension study achieved clinical remission, per their partial Mayo score. ${ }^{26}$

\section{Adalimumab in ulcerative colitis: real-life data}

Although adalimumab has been recently licensed, multiple lines of evidence from open-label and retrospective studies on adalimumab, administered for compassionate use in ulcerative colitis patients, have been available for several years (Table 1). Oussalah et $\mathrm{al}^{27}$ first presented data on 13 ulcerative colitis patients treated with adalimumab in 2008. All of the patients had been previously treated with infliximab, and most of them $(90.31 \%)$ had been previously treated with thiopurines. Patients were treated with adalimumab, with an induction dose of 160/80 mg at weeks 0 and 2, and then maintained with $40 \mathrm{mg}$ EOW. The primary endpoint was defined as the proportion of patients on adalimumab therapy during the study. After a median follow-up of 41 weeks, the percentage of patients remaining on adalimumab therapy was $32.5 \%$. Eight patients discontinued adalimumab: six due to colectomy, one due to lack of response, and one due to an exacerbation of psoriasis. No significant differences were found in adalimumab withdrawal and colectomy rates between the patients who lost response to infliximab and those who became intolerant. From this small cohort of difficult-to-treat patients who had already been treated with all of the main available therapies, adalimumab treatment potentially avoided colectomy in about half of them.

One year later, the Mayo Clinic Group published the results of an uncontrolled, open-label study on adalimumab in 20 patients with ulcerative colitis, of whom $35 \%$ were naïve to infliximab. All patients had active disease (defined as a Mayo score of 6-12 points, with an endoscopic subscore of at least 2) despite concurrent treatment (steroids, and/or thiopurines, and/or aminosalicylates). Patients were treated with adalimumab at an induction dose of 160/80 $\mathrm{mg}$ at weeks 0 and 2, respectively, and maintained with $40 \mathrm{mg}$ EOW. The primary endpoint was defined as the proportion of patients achieving a clinical response at week 8 . The percentages of patients who had a clinical remission or response were $5 \%$ and $25 \%$ at week 8 , respectively and $25 \%$ and $50 \%$ at week 24 , respectively. No significant differences were found between infliximab-naïve and infliximab-exposed patients. Among the patients who entered the trial on corticosteroids, $58 \%$ were able to withdraw by week 24 , showing the potential effectiveness of adalimumab as a steroid-sparing agent. ${ }^{28}$

Hudis et a ${ }^{29}$ retrospectively reported data on nine patients, with active ulcerative colitis (mean Mayo score of $6 \pm 1.66$ at baseline) and secondary infliximab failure, who were treated with adalimumab (induction, $160 / 80 \mathrm{mg}$ at weeks $0 / 2$; maintenance, $40 \mathrm{mg}$ EOW). During the follow-up, 
adalimumab was found to be effective at inducing a clinical response (mean Mayo score, $2.5 \pm 1)(P<0.0005)$, with a steroid-sparing effect $(P<0.003)$.

Gies et al, ${ }^{30}$ in 2010, also reported "real life" data, from a single referral center, involving 53 ulcerative colitis outpatients treated with biologic drugs, following a structured protocol for a "step-up" approach. All patients were intolerant and/or nonresponders to conventional therapy, including aminosalicylates, steroids, and thiopurines. Among them, 25 patients were treated with adalimumab (160/80 $\mathrm{mg}$ at weeks 0 and 2, then $40 \mathrm{mg}$ EOW) and 28 with infliximab ( $5 \mathrm{mg} / \mathrm{kg}$ at weeks 0,2 , and 6 , then every 8 weeks). Most of patients had extensive colitis $(96 \%$ in the adalimumab group and $90 \%$ in the infliximab group). Concomitant immunosuppressive therapy (azathioprine or methotrexate) was taken by significantly fewer patients in the adalimumab group (20\% vs 53.4\%) ( $P=0.0118)$. The primary endpoint was defined as the proportion of patients treated with adalimumab or infliximab achieving and maintaining a clinical response. At 14 weeks, 47 of the $53(88.7 \%)$ patients had a clinical response to anti-TNF therapy, without a significant difference between the adalimumab (20/25 patients, $80 \%)$ and infliximab $(27 / 28$ patients, $96 \%)$ groups $(P=0.0889)$. Among the patients who entered the maintenance treatment phase, $14 / 20$ adalimumab (70.0\%) and 14/18 (77.8\%) infliximab patients had a response up to the end of follow-up $(P=0.7190)$. The median duration for the maintenance phase was 54.5 weeks (range, 3-108 weeks) for the adalimumab group and 64.5 weeks (range, 8-180 weeks) for the infliximab group. Of the six adalimumab patients who lost response, two underwent colectomy, one switched to infliximab, and three were treated with another course of steroids. About $92 \%$ of the patients who were initially taking steroids were able to stop over the course of the maintenance period, with similar results observed in both groups. Thus, in this "real life" cohort, adalimumab seemed to be as effective as infliximab at achieving induction and maintenance responses in ulcerative colitis patients.

The effectiveness of adalimumab in a real-life setting has also been reported in a Spanish retrospective multicenter study that enrolled 30 ulcerative colitis patients after the failure of other therapies. All patients were infliximab experienced: $53.3 \%$ had lost responsiveness, $40 \%$ had become intolerant, and $6.7 \%$ were primary nonresponders. Adalimumab was administered to 26 patients because of moderate-to-severe, refractory ulcerative colitis, and four patients received adalimumab because of a severe attack that was refractory to intravenous corticosteroids. All patients received a loading dose of 160/80 mg of adalimumab at weeks 0 and 2, respectively and were maintained with $40 \mathrm{mg}$ EOW. Patients were assessed at weeks 4 and 12, and then every 4 weeks in order to evaluate the short- and long-term outcomes. The primary endpoint was defined as the induction of a clinical response at week 12 . Sixteen $(53.3 \%)$ and $18(60 \%)$ patients achieved a clinical response at week 4 and 12, respectively. Three (10\%) and eight (26.7\%) patients achieved a clinical remission at week 4 and 12, respectively. Fifteen (50\%) patients discontinued adalimumab during the median follow-up of 48 weeks (interquartile range [IQR], 16-104), and $13(86.6 \%)$ of them discontinued because of a lack or loss of response, including four inpatients with severe intravenous corticosteroid-refractory disease. All patients who were under corticosteroid treatment at baseline and entered the maintenance adalimumab treatment were able to discontinue the steroids. The rate of colectomy was $20 \%$, with a median time to colectomy of 16 weeks (IQR, 5.2-40.5 weeks). A lack of response at week 12 was associated with an increased probability of withdrawal $(P=0.001)$ and a higher rate of colectomy $(P=0.001)$. Thus, adalimumab induced a durable clinical response in a good percentage of patients with medically refractory ulcerative colitis, especially in those who achieved short-term clinical response. ${ }^{31}$

The same issue was also addressed by McDermott et al, ${ }^{32}$ who collected data on 23 patients with ulcerative colitis treated with adalimumab (standard induction and maintenance treatment). Twenty-two of the patients (96\%) had received prior immunomodulatory therapy and 20 (86\%) had previously been treated with infliximab (three primary nonresponders, eleven secondary failures, and six who experienced side effects). The primary endpoint was defined as treatment failure. During a median follow-up period of 22 months (IQR, 8-32 months), 16 patients (69.5\%) discontinued adalimumab. The reasons for discontinuation were primary nonresponse in six patients (37\%), secondary nonresponse in eight (50\%), and side effects in one $(6 \%)$. Nine patients underwent colectomy, and three refused surgery; the colectomy-free survival was estimated to be $78 \%$ at 6 months, $70 \%$ at 12 months, and $59 \%$ at 2 years. No significant predictors of colectomy were identified, but $55 \%$ of patients who underwent surgery had failed adalimumab treatment within 3 months of starting treatment. $^{32}$

Further findings by Ferrante et $\mathrm{al}^{33}$ confirmed that adalimumab is effective in inducing a durable clinical remission in patients who have already been treated with infliximab. Fifty patients with moderate-to-severe ulcerative colitis received adalimumab induction treatment $(160 / 80 \mathrm{mg}$ at weeks 0 
and 2, followed by $40 \mathrm{mg}$ EOW). The primary endpoint was the long-term efficacy of adalimumab. At week $4,68 \%$ patients showed a short-term clinical response. In particular, $22 \%$ of the patients achieved a complete clinical response, defined as the absence of diarrhea and bloody stools, and $46 \%$ of the patients achieved a partial response, defined as a marked clinical improvement, with persisting rectal blood loss. After a median follow-up of 23 months, 52\% achieved a durable response to adalimumab, defined as a lasting clinical response. Colectomy was necessary in $20 \%$ of patients. Dose escalation was necessary in $76 \%$ of patients and was associated with significantly increased serum adalimumab levels (from 4.75 to $7.95 \mu \mathrm{g} / \mathrm{mL})(P=0.023)$. Short-term clinical response and response to dose escalation were associated with colectomy-free survival $(P=0.030$ and $P<0.001$, respectively).

Data from the Spanish ENEIDA (Estudio Nacional en Enfermedad Inflamatoria Intestinal sobre Determinantes genéticos y Ambientales) registry of 48 patients with ulcerative colitis treated with adalimumab (induction dose, $160 / 80 \mathrm{mg}$ at weeks 0 and 2 , in $93.7 \%$ of patients; maintenance dose, $40 \mathrm{mg}$ EOW) have been recently reported. ${ }^{31}$ Among these patients, 39 (81.3\%) had previously received infliximab and were categorized into one of three categories: remission, 51.3\%; response, 33.3\%; and primary nonresponse, $15.4 \%$. The primary endpoint was defined as the proportion of patients achieving a clinical response during the follow-up period. The clinical response rates were assessed at weeks 12,28 , and 54 and were $70.8 \%$ (34/48), 43.2\% (19/44), and 35\% (14/40), respectively. A response to prior treatment with infliximab was the only factor predictive of a response to adalimumab at week 12 , which was obtained in $90 \%$ of infliximab remitters, $53.8 \%$ of responders, and $33.3 \%$ of primary nonresponders $(P=0.01)$. Eleven patients $(22.9 \%)$ needed colectomy after a mean time of 205 days. A lack of response to adalimumab at week 12 was shown to be an independent predictor of colectomy: five of the 34 (14.7\%) responders and six of the $14(42.9 \%)$ nonresponders $(P=0.035)$ required a colectomy, with a colectomy-free time that was significantly reduced among the nonresponding patients $(P=0.01) .{ }^{34}$

The last real-life experience comes from an Italian multicenter study that represents the largest case series of active ulcerative colitis treated with adalimumab. Eighty-eight patients were treated with adalimumab (induction dose of $160 \mathrm{mg} / 80 \mathrm{mg}$ in 77 patients [87.5\%] and $80 \mathrm{mg} / 40 \mathrm{mg}$ in eleven patients [12.5\%], at weeks 0 and 2, respectively). After induction, the patients were maintained with adalimumab
40 mg EOW; a dose escalation was allowed at the physician's discretion. All patients had active disease (medium partial Mayo score, 6) (IQR, 4-8), and 57 (64.8\%) had extensive colitis. Sixty-nine patients $(78.4 \%)$ had been previously treated with infliximab, and 65 (73.9\%) had been exposed to immunomodulators (azathioprine, methotrexate, and cyclosporine). The indications for adalimumab treatment were corticosteroid dependence in 41 patients (46.6\%), corticosteroid resistance in 23 patients (26.1\%), extraintestinal manifestations in 14 patients (15.9\%), and a combination of corticosteroid dependence and extraintestinal manifestations in ten patients (11.4\%). The median duration of adalimumab therapy was 13 months (IQR, 6-21 months), with a median follow-up duration of 15.5 months (IQR, 12-24 months). The co-primary endpoints were defined as the proportion of patients achieving clinical remission (partial Mayo score $\leq 1$ ) at $4,12,24$, and 54 weeks. The clinical remission rates were $17 \%, 28.4 \%, 36.4 \%$, and $43.2 \%$ at $4,12,24$, and 54 weeks, respectively, with no significant differences between the infliximab-naïve and infliximab-exposed patients. Fifteen patients (17\%) achieved sustained clinical remission, defined as a lasting clinical remission from week 12 up to 24 and 54 weeks. Among the 60 patients who were taking steroids at baseline, $56.7 \%$ were able to discontinue steroids, and a steroid-free remission was achieved in 24 of 60 patients $(40.0 \%)$ at week 54. Fifty-seven patients underwent baseline and follow-up endoscopy after a median of 11 months (IQR, 5.1-13.2 months). An endoscopic remission was achieved in $28(49.1 \%)$ of 57 patients, and $15(26.3 \%)$ of the 57 achieved complete mucosal healing. Overall, 25\% (22 of 88) of the patients required colectomy, with a median time to colectomy of 5.5 months (IQR, 3-13 months). The rate of colectomy was higher in the infliximab-exposed group than in the infliximab-naïve group (28.9\% vs $10.5 \%)$, but this result did not achieve statistical significance, probably because of the small number of patients who ultimately required surgery and the small number of infliximab-naïve patients enrolled in the study. In conclusion, in this large, real-life cohort of refractory and difficult-to-treat ulcerative colitis patients, adalimumab was shown to be effective at inducing and maintaining a durable clinical remission and to have a steroid-sparing effect. ${ }^{35}$

\section{Safety}

Adalimumab treatment is generally well tolerated. The subcutaneous administration is associated with generally mild injection site reactions that do not necessitate drug discontinuation. The overall safety profile in the clinical 
trials in patients with ulcerative colitis was comparable to that of placebo, and the rates of adverse events were similar to the ones that emerged for the other approved indications for adalimumab. ${ }^{36}$ No relevant warnings have emerged from the real-life studies. However, in two studies, a worsening of preexisting psoriasis, leading to drug withdrawal, was recorded. ${ }^{24,28}$ Lastly, the fully human-designed features of adalimumab reduce, but do not eliminate, the risk of antidrug antibody development. ${ }^{22}$ The presence of human anti-human antibodies (HAHA) and low trough serum adalimumab levels have been reported to influence the long-term outcome of adalimumab therapy in patients with Crohn's disease. Increased adalimumab discontinuation rates have also been reported in Crohn's disease patients with low trough serum adalimumab concentrations. Furthermore, HAHA were detected in $92 \%$ of Crohn's disease patients with low trough serum adalimumab levels during follow-up, probably reflecting the increased clearance of the drug and the subsequent loss of response. Finally, concomitant immunosuppressive agents did not affect treatment outcomes, adalimumab trough levels, or HAHA development. ${ }^{37}$

\section{Conclusion}

The treatment of ulcerative colitis depends largely on the extension, severity, and behavior of the disease, and the traditional step-up approach with conventional drugs remains the standard management approach. However, there are subsets of patients who do not respond to conventional therapies or in whom conventional therapies are contraindicated. In these difficult-to-treat patients, the current guidelines recommend the use of biological drugs. In the recent past, infliximab was the only biological drug approved for the treatment of patients with ulcerative colitis. Recently, after the publication of the results of the two pivotal, randomized ULTRA 1-2 trials, adalimumab was approved for use in patients with moderately-to-severely active disease, nonresponders, and those intolerant to conventional therapy. As often happens, the results of a clinical trial should be incorporated into real-life clinical practice, where patients have already experienced several therapies before, and may be intolerant or not fully adherent to treatments. Therefore, the goal is to select the candidates who will best benefit from the drug. The emerging concept is that the ideal candidates for adalimumab therapy are anti-TNF $\alpha$-naïve outpatients with moderateto-severe corticosteroid- or immunosuppressive-refractory ulcerative colitis. Short-term clinical responses, evaluated after 8-12 weeks, seem to influence the long-term outcomes and are associated with more durable clinical responses and colectomy-free survival. Adalimumab also demonstrated a steroid-sparing effect and a mucosal healing capacity, and it may be a valid option for steroid-dependent patients. As has now been demonstrated in pivotal trials and in several real-life experiences, adalimumab is effective and safe for treating patients with different types of ulcerative colitis, including difficult-to-treat individuals.

\section{Disclosure}

AA received consultancy from AbbVie and MSD; lecture fees from AbbVie, MSD, Chiesi, Ferring, Nycomed, and Otsuka; and educational grants from AbbVie and MSD. LG received educational grants from AbbVie, MSD. The authors report no other conflicts of interest.

\section{References}

1. Dignass A, Eliakim R, Magro F, et al. Second European evidence-based consensus on the diagnosis and management of ulcerative colitis part 1: definitions and diagnosis. J Crohns Colitis. 2012;6(10):965-990.

2. Silverberg MS, Satsangi J, Ahmad T, et al. Toward an integrated clinical, molecular and serological classification of inflammatory bowel disease: Report of a Working Party of the 2005 Montreal World Congress of Gastroenterology. Can J Gastroenterol. 2005;19 Suppl A:5-36.

3. Ekbom A, Helmick C, Zack M, Adami HO. Ulcerative colitis and colorectal cancer. A population-based study. N Engl J Med. 1990; 323(18):1228-1233.

4. Solberg IC, Lygren I, Jahnsen J, et al; IBSEN Study Group. Clinical course during the first 10 years of ulcerative colitis: results from a population-based inception cohort (IBSEN Study). Scand J Gastroenterol. 2009;44(4):431-440.

5. D'Haens G, Sandborn WJ, Feagan BG, et al. A review of activity indices and efficacy end points for clinical trials of medical therapy in adults with ulcerative colitis. Gastroenterology. 2007;132(2):763-786.

6. Ford AC, Achkar JP, Khan KJ, et al. Efficacy of 5-aminosalicylates in ulcerative colitis: systematic review and meta-analysis. $\mathrm{Am} J$ Gastroenterol. 2011;106(4):601-616.

7. Dignass A, Lindsay JO, Sturm A, et al. Second European evidence-based consensus on the diagnosis and management of ulcerative colitis part 2: current management. J Crohns Colitis. 2012;6(10):991-1030.

8. Rutgeerts P, Sandborn WJ, Feagan BG, et al. Infliximab for induction and maintenance therapy for ulcerative colitis. $N$ Engl J Med. 2005; 353(23):2462-2476.

9. Ardizzone S, Maconi G, Russo A, Imbesi V, Colombo E, Bianchi Porro G. Randomised controlled trial of azathioprine and 5-aminosalicylic acid for treatment of steroid dependent ulcerative colitis. Gut. 2006; 55(1):47-53.

10. Panaccione R, Rutgeerts P, Sandborn WJ, Feagan B, Schreiber S, Ghosh S. Review article: treatment algorithms to maximize remission and minimize corticosteroid dependence in patients with inflammatory bowel disease. Aliment Pharmacol Ther. 2008;28(6):674-688.

11. Armuzzi A, Pugliese D, Danese S, et al. Infliximab in steroid-dependent ulcerative colitis: effectiveness and predictors of clinical and endoscopic remission. Inflamm Bowel Dis. Epub February 27, 2013.

12. Laharie D, Bourreille A, Branche J, et al; Groupe d'Etudes Thérapeutiques des Affections Inflammatoires Digestives. Ciclosporin versus infliximab in patients with severe ulcerative colitis refractory to intravenous steroids: a parallel, open-label randomised controlled trial. Lancet. 2012;380(9857):1909-1915.

13. Moskovitz DN, Van Assche G, Maenhout B, et al. Incidence of colectomy during long-term follow-up after cyclosporine-induced remission of severe ulcerative colitis. Clin Gastroenterol Hepatol. 2006;4(6):760-765. 
14. HUMIRA $^{\circledR}$ (adalimumab) subcutaneous injection [product monograph]. North Chicago, IL: Abbott Laboratories, Ltd; 2012.

15. Hanauer SB, Sandborn WJ, Rutgeerts P, et al. Human anti-tumor necrosis factor monoclonal antibody (adalimumab) in Crohn's disease: the CLASSIC-I trial. Gastroenterology. 2006;130(2):323-333.

16. Colombel JF, Sandborn WJ, Rutgeerts P, et al. Adalimumab for maintenance of clinical response and remission in patients with Crohn's disease: the CHARM trial. Gastroenterology. 2007;132(1):52-65.

17. Sandborn WJ, Rutgeerts P, Enns R, et al. Adalimumab induction therapy for Crohn disease previously treated with infliximab: a randomized trial. Ann Intern Med. 2007;146(12):829-838.

18. Panaccione R, Colombel JF, Sandborn WJ, et al. Adalimumab sustains clinical remission and overall clinical benefit after 2 years of therapy for Crohn's disease. Aliment Pharmacol Ther. 2010;31(12):1296-1309.

19. Colombel JF, Schwartz DA, Sandborn WJ, et al. Adalimumab for the treatment of fistulas in patients with Crohn's disease. Gut. 2009; 58(7):940-948.

20. Reinisch W, Sandborn WJ, Hommes DW, et al. Adalimumab for induction of clinical remission in moderately to severely active ulcerative colitis: results of a randomised controlled trial. Gut. 2011;60(6):780-787.

21. Sandborn WJ, van Assche G, Reinisch W, et al. Adalimumab induces and maintains clinical remission in patients with moderate-to-severe ulcerative colitis. Gastroenterology. 2012;142(2):257-265.

22. Reinisch W, Sandborn WJ, Kumar A, Pollack PF, Lazar A, Thakkar RB. 52 -week clinical efficacy with adalimumab in patients with moderately to severely active ulcerative colitis who failed corticosteroids and/or immunosuppresants. Gut. 2011;60 Suppl 1:A139-A140.

23. Sandborn WJ, Colombel JF, D'Haens G, et al. One-year maintenance outcomes among patients with moderately-to-severely active ulcerative colitis who responded to induction therapy with adalimumab: subgroup analyses from ULTRA 2. Aliment Pharmacol Ther. 2013;37(2): 204-213.

24. Feagan BG, Sandborn WJ, Thakkar RB, et al. Adalimumab induction dose reduces the risk of hospitalizations and colectomies in patients with ulcerative colitis during the first 8 weeks of therapy. Gut. 2012; 61 Suppl 3:A283.

25. Feagan BG, Sandborn WJ, Skup M, et al. Adalimumab therapy reduces hospitalization and colectomy rates in patients with ulcerative colitis among initial responders. Gut. 2012;61 Suppl 3:A164.

26. Colombel JF, Sandborn WJ, Wolf D, et al. Long term efficacy of adalimumab for treatment of moderate to severe ulcerative colitis. Gut. 2012;61 Suppl 3:A80
27. Oussalah A, Laclotte C, Chevaux JB, et al. Long-term outcome of adalimumab therapy for ulcerative colitis with intolerance or lost response to infliximab: a single-centre experience. Aliment Pharmacol Ther. 2008;28(8):966-972.

28. Afif W, Leighton JA, Hanauer SB, et al. Open-label study of adalimumab in patients with ulcerative colitis including those with prior loss of response or intolerance to infliximab. Inflamm Bowel Dis. 2009; 15(9):1302-1307.

29. Hudis N, Rajca B, Polyak S, Zeilman CJ, Valentine JF. The outcome of active ulcerative colitis treated with adalimumab. Gastroenterology. 2009;136(5 Suppl 1):A661.

30. Gies N, Kroeker KI, Wong K, Fedorak RN. Treatment of ulcerative colitis with adalimumab or infliximab: long-term follow-up of a singlecentre cohort. Aliment Pharmacol Ther. 2010;32(4):522-528.

31. Taxonera C, Estellés J, Fernández-Blanco I, et al. Adalimumab induction and maintenance therapy for patients with ulcerative colitis previously treated with infliximab. Aliment Pharmacol Ther. 2011;33(3):340-348.

32. McDermott E, Murphy S, Keegan D, O'Donoghue D, Mulcahy H, Doherty G. Efficacy of adalimumab as a long term maintenance therapy in ulcerative colitis. J Crohns Colitis. 2013;7(2):150-153.

33. Ferrante M, Karmiris K, Compernolle G, et al. Efficacy of adalimumab in patients with ulcerative colitis: restoration of serum levels after dose escalation results in a better long-term outcome. Gut. 2011; 60 Suppl 3:A72.

34. García-Bosch O, Gisbert JP, Cañas-Ventura A, et al. Observational study on the efficacy of adalimumab for the treatment of ulcerative colitis and predictors of outcome. J Crohns Colitis. 2012. Epub November 8, 2012.

35. Armuzzi A, Biancone L, Daperno M, et al. Adalimumab in active ulcerative colitis: a "real-life" observational study. Dig Liver Dis. In press 2013.

36. Burmester GR, Mease P, Dijkmans BA, et al. Adalimumab safety and mortality rates from global clinical trials of six immune-mediated inflammatory diseases. Ann Rheum Dis. 2009;68(12):1863-1869.

37. Karmiris K, Paintaud G, Noman M, et al. Influence of trough serum levels and immunogenicity on long-term outcome of adalimumab therapy in Crohn's disease. Gastroenterology. 2009;137(5):1628-1640.
Drug Design, Development and Therapy

\section{Publish your work in this journal}

Drug Design, Development and Therapy is an international, peerreviewed open-access journal that spans the spectrum of drug design and development through to clinical applications. Clinical outcomes, patient safety, and programs for the development and effective, safe, and sustained use of medicines are a feature of the journal, which

\section{Dovepress}

has also been accepted for indexing on PubMed Central. The manuscript management system is completely online and includes a very quick and fair peer-review system, which is all easy to use. Visit http://www.dovepress.com/testimonials.php to read real quotes from published authors. 